\title{
The effect of camera temperature variations on stereo-digital image correlation measurements
}

\author{
Bing Pan, ${ }^{1,}{ }^{*}$ Wentao ShI, ${ }^{1}$ and Gilles Lubineau ${ }^{2}$ \\ ${ }^{1}$ Institute of Solid Mechanics, Beihang University, Beijing 100191, China \\ ${ }^{2}$ King Abdullah University of Science and Technology (KAUST), Physical Science and Engineering Division. COHMAS laboratory, Thuwal \\ 23955-6900, Saudi Arabia \\ *Corresponding author: panb@buaa.edu.cn
}

Received XX Month XXXX; revised XX Month, XXXX; accepted XX MonthXXXX; posted XX Month XXXX (Doc. ID XXXXX); publishedXX Month XXXX

In laboratory and especially non-laboratory stereo-digital image correlation (stereo-DIC) applications, the extrinsic and intrinsic parameters of the cameras used in a stereo-DIC system may change slightly due to the camera warm-up effect and the possible variations in ambient temperature. Because these camera parameters are generally calibrated once prior to measurements and considered to be unaltered during measurement, the changes in these parameters unavoidably induce displacement/strain errors. In this study, the effect of temperature variations on stereo-DIC measurements is investigated experimentally. To quantify the errors associated with camera or ambient temperature changes, surface displacements and strains of a stationary optical quartz glass plate with near-zero thermal expansion were continuously measured using a stereo-DIC system. The results confirm that: (1) temperature variations in the cameras and ambient environment have a considerable influence on the displacements and strains measured by stereo-DIC because of the slightly altered extrinsic and intrinsic camera parameters; (2) the corresponding displacement and strain errors correlate with temperature changes. For the specific stereo-DIC configuration used in this work, the temperature-induced strain errors were estimated to be approximately $30-50 \mu \varepsilon /{ }^{\circ} \mathrm{C}$. To minimize the adverse effect of temperature variations on stereo-DIC measurements, simple but effective solutions are suggested.

OCIS codes: (120.0120) Instrumentation, measurement, and metrology; (110.0110) Imaging systems; (120.3940) Metrology.

http://dx.doi.org/10.1364/AO.99.099999

\section{Introduction}

Stereo digital image correlation (Stereo-DIC) technique (also known as 3D-DIC) has been widely used for shape, motion and deformation measurements in various scientific research and engineering applications [1-5]. By comparing the image pairs of a test object surface recorded at different configurations using two synchronized digital cameras with pre-calibrated camera parameters, stereo-DIC technique can simultaneously determine all the three displacement components of a test object surface by subtracting the eorrdinates of corresponding 3D point clouds. Compared with other interferometric optical techniques for shape and 3D deformation measurement, the stereo-DIC technique features the prominent merits of simple experimental setup and easy implementation procedure, low requirement on ambient environment and wider range of usability. Numerous applications of stereo-DIC have successful demonstrated its practicality and versatility. Commercial stereo-DIC systems [6-8] with powerful capacities are also available in market.

To achieve high-accuracy measurements, various aspects involved in the implementation of stereo-DIC must be given careful consideration. In the literature, robust, efficient and accurate subset-based image matching algorithms and corresponding bias error correction approaches have been proposed to register the same physical points imaged in different views and various configurations [9-13]. Advanced stereo-calibration techniques using bundle adjustment have also been advocated by various researchers [14,15] with 
claimed reprojection errors smaller than 0.001pixels. In addition, an active imaging stereo-DIC system, which combines an actively illuminated monochromatic lighting and bandpass filter imaging, has also been proposed for deformation measurement at non-laboratory conditions or extreme high-temperature environments [4] without being affected by ambient light variations. However, a fundamental assumption implicitly made in all these research efforts for achieving high-accuracy 3D deformation measurement is the supposed stability of a stereo-DIC system [16,17], which assumes that, once a stereo-DIC system is calibrated, the resulting extrinsic and intrinsic parameters will not change during the followed measurement period.

In fact, although strong mechanical fixation can ensure the stability of the two cameras in terms of relative orientation and position in a stereo-DIC system, the intrinsic geometry of two cameras may change slightly due to the warm-up effect of cameras and ambient temperature variations. Actually, the slight temperature-induced change in camera and its effect on single camera-based optical techniques [18-21] for motion tracking and strain mapping have been noticed for a long time. For instance, Handel [18] observed that image shifts to some tenth of a pixel after camera start-up, and developed a mode to explain and compensate for image drift. Ma et al. [19] and Pan et al. [20] demonstrated that for the single camera 2D-DIC, the self-heating of digital cameras can give rise to strain errors to an extent of $70-230 \mu \varepsilon$ depending on the specific cameras. More recently, Yu et al. [21] studied the effect of temperature variations on the videometrics measurement. They presented first an image drift model and then a camera parameter-temperature model to compensate for temperate-induced image drifts. Regrettably, as for the more powerful and practical stereo-DIC technique, the influences of temperature variation on stereo-DIC measurements have not yet been reported. Since the accuracy of stereo-DIC measurements is always the most important requirement in experimental mechanics applications, quantifying and minimizing the adverse effect of temperature variations on stereo-DIC measurements should be helpful to the optimal use of this powerful technique.

In this work, two sets of experiments were designed to investigate the effect of temperature variations on stereo-DIC measurements. The experimental setup and experimental procedures are detailed in section 2. The resulting displacements and strains due to temperature variations are analyzed and discussed in section 3. Conclusions and corresponding suggestions to minimize the adverse effect of temperature variations on the stereo-DIC measurements are summarized in Section 4.

\section{Study of the effect of temperature variations on stereo-DIC measurements}

\section{A. Experimental setup and the test specimen}

The experimental setup used to investigate the effect of temperature variations on stereo-DIC measurements is shown in Fig. 1. The stereo-DIC system used in this work comprises two industrial cameras (GRAS-50S5M-C, Point Grey Research, Inc.) with a spatial resolution of $2448 \times 2048$ pixels at 256 gray levels. Each camera equipped with a $50 \mathrm{~mm}$ fixed-focus optical lens (Jos. Schneider Optische Werke GmbH, Germany). Note that to eliminate the adverse influence of potential ambient light variations on the DIC measurement [4], two optical bandpass filters, with a center wavelength of $450 \pm 2 \mathrm{~nm}$ and a full-width at half-maximum value of $32 \mathrm{~nm}$, were attached just before the two lenses. Also, to provide even and stable active illumination, a self-made monochromatic blue LED light source, emitting at 450-455 $\mathrm{nm}$, employed to illuminate the test object surface.

An optical grade quartz glass plate $(\mathrm{w} \times \mathrm{h} \times \mathrm{t}=70 \mathrm{~mm} \times 70 \mathrm{~mm} \times 5 \mathrm{~mm})$ was used as the reference sample. Random speckle patterns were decorated onto the sample surface by first spraying a thin layer of white paints and then black paints. The quartz plate with premade speckle patterns was then fixed and kept stationary during the following tests. Since the coefficient of thermal expansion of the quartz plate is close to zero, this sample, with near-zero thermal expansion, can be seen as a stationary rigid reference object with zero displacements and strains. As a consequence, the non-zero displacements and strains on the sample surface detected by the stereo-DIC can only be regarded as the virtual displacements and strains caused by the instability of the stereo-DIC system.

In the following tests, the stereo-DIC system was placed about $730 \mathrm{~mm}$ in the front of the test sample with the two cameras aligned at a proper angle to ensure the region of interest can be fully imaged by both cameras. To record the temperature changes in the specimen and the two cameras, three thermocouple temperature sensors with a reading 
accuracy of $0.01^{\circ} \mathrm{C}$ were used with one sensor being attached onto the backside of the sample, two others being bonded to the two cameras, as shown in Fig.1.

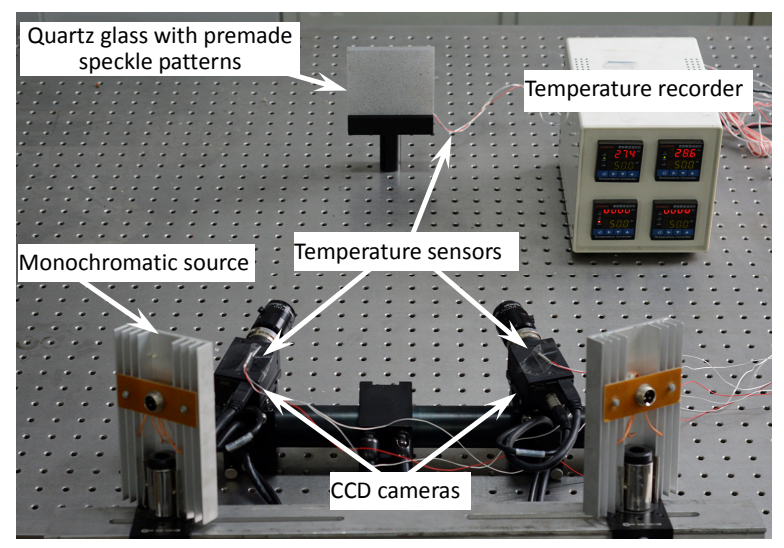

Fig. 1. Experimental setup for examining the effect of temperature variations on stereo-DIC measurements

\section{B. Experimental procedure}

In this study, two sets of experiments were designed to investigate the effect of temperature variations in cameras and ambient environments, respectively, on stereo-DIC measurements. In the first set of experiment, the ambient temperature was assumed to be constant, which is characteristic for most indoor stereo-DIC measurements. We only examined the impact of temperature changes after the startup of cameras on DIC measurements. Prior to the image recoding process, the optical lenses were carefully adjusted to get a clear image of the test sample surface. Then, the experiments comprise the following steps:

1) Capture 30 calibration image pairs of a calibration target at different positions or orientations after the tartup of the stereo-DIC system. These image pairs are subsequently used to calibrate the intrinsic and extrinsic parameters of stereo-DIC system in the original state before achieving thermal equilibrium;

2) Record speckle image pair of the quartz plate every 2 minutes, and in the meantime record the temperature data of the two camera's outer case and the target board. The image and temperature recording process lasted for 5 hours, resulting in a total of 150 image pairs of the sample surface and corresponding temperature data;

3) Take 30 image pairs of the same calibration target after imaging recording procedure. These image pairs are used to determine the intrinsic and extrinsic camera parameters after reaching thermal equilibrium.

The second set of experiment was designed to analyze the ambient temperature changes in out-door environments on the stereo-DIC measurements. In the experiments, before image recording, the two cameras had been preheated for two hours and were assumed to have reached their thermal balance. Then the stereo-DIC system was calibrated using the same calibration procedure described above. Next, the ar ıbient temperature was changed by turning on and turning r,ff an air conditioner. At the meantime, the image pair of the specimen surface and the corresponding temperatures of the two cameras and the specimen surface were recorded every $2 \min$.

\section{Correlation analysis}

The recorded calibration image pairs and experimental image pairs were subsequently processed using the commercial software PMLAB DIC-3D to determine the extrinsic and intrinsic parameters of the two cameras, and also to calculate the 3D displacement fields of the quartz sample surface. It should be noted that, during correlation analysis, the initially recorded image pair is used as the reference image. Figure 2 shows the left and right images of the test sample recorded as the reference image pair. A rectangle ROI of $1840 \times 1240$ pixels comprising 5859 (=93 rows $\times 63$ columns) calculation points (grid step $=20$ pixels) is defined in the image captured by the left camera. The displacements of these calculation points in other images are tracked by optimizing the zero-mean normalize sum of squared difference (ZNSSD) criterion using inverse compositional Gauss-Newton algorithm $[9,10]$ with a subset size of $101 \times 101$ pixels. After retrieving the displacement fields, the strain maps can be further calculated by using pointwise local least square method [22] with a strain window of $15 \times 15$ points (corresponding to a local area of $281 \times 281$ pixels). Also, mean displacements and strains of the ROI can be calculated by taking the average values of the displacement and strain maps.

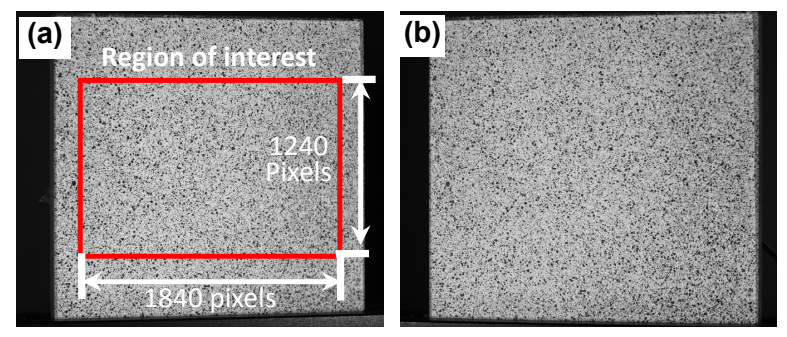


Fig. 2. Captured reference image pair in the self-heating tests: (a) left view; (b) right view

\section{Results and discussion}

\section{A. Self-heating tests}

Figure 3 shows typical temperature-time curves recorded by the three temperature sensors in the self-heating tests. Since this test was performed indoor, the temperatures of the test sample remain approximately constant with small variations lying between $19^{\circ} \mathrm{C}$ and $20^{\circ} \mathrm{C}$, whereas the temperatures on the two camera bodies increased about $9^{\circ} \mathrm{C}$ after startup. Also, it is found that the two camera bodies' temperatures increased rapidly in the first 1.5 hour which is known as warm-up stage. Then the temperatures remain almost stable and achieve the so-called thermal equilibrium stage. These observations show good agreement with reports given in existing works [17-19].

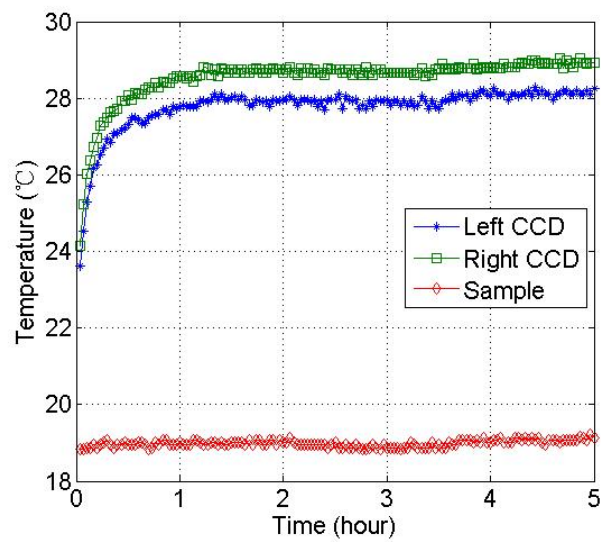

Fig. 3. The detected temperature variations of two cameras and the quartz glass plate

Using the two sets of calibration image pairs recorded at two different configurations (one set after just opening the cameras, and the other set after reaching thermal equilibrium), the camera parameters can be determined. The calibrated intrinsic and extrinsic parameters of the two cameras of the stereo-DIC system in these initial and final states are listed in Table 1, where $\left(c_{x}, c_{y}\right)$ are the coordinates of principle points of the cameras, $f$ is the effective length in units of pixels, $k_{1}$ are the first-order radial distortion coefficients of the lenses. $\left(t_{x}, t_{y}, t_{z}\right)(m m)$ are the position of pinhole in camera 2 relative to camera $1 ;(\alpha, \beta, \gamma)$ (degrees) are the relative orientation of right camera with respect to left camera, where $\alpha$ is the relative tilt, $\beta$ the relative pan angle, and $\gamma$ the swing angle. The relative percentage errors listed in the last column show the slight changes due to temperature-induced changes in the intrinsic geometry of the two cameras.

Table 1 Calibration parameters of 3D-DIC measurement system at before and after achieving thermal equilibrium

\begin{tabular}{|c|c|c|c|c|}
\hline \multicolumn{2}{|c|}{ Camera parameters } & \multirow{2}{*}{$\begin{array}{c}\text { Initial } \\
\text { state } \\
1305.12\end{array}$} & \multirow{2}{*}{$\begin{array}{c}\text { Final state } \\
1302.69\end{array}$} & \multirow{2}{*}{$\begin{array}{c}\text { Relative } \\
\text { error (\%) }\end{array}$} \\
\hline \multirow{4}{*}{$\begin{array}{c}\text { Left } \\
\text { camera }\end{array}$} & $c x($ pixel $)$ & & & \\
\hline & $c y($ pixel $)$ & 1025.32 & 1024.28 & -0.00101432 \\
\hline & $f($ pixel $)$ & 16049.3 & 16029.5 & -0.00123370 \\
\hline & $k_{1}$ & 0.0661621 & 0.054812 & -0.171549 \\
\hline \multirow{4}{*}{$\begin{array}{l}\text { Right } \\
\text { camera }\end{array}$} & $c x($ pixel $)$ & 1247.7 & 1105.16 & -0.114242 \\
\hline & $c_{y}($ pixel $)$ & 993.532 & 967.789 & -0.0259106 \\
\hline & $f($ pixel $)$ & 15823.5 & 16276.8 & 0.0286473 \\
\hline & $k_{1}$ & 0.0697796 & 0.0362887 & -0.479952 \\
\hline \multirow{7}{*}{$\begin{array}{c}\text { Transform } \\
\text { ation }\end{array}$} & $\alpha(\operatorname{deg})$ & -0.875953 & -0.945546 & 0.0794483 \\
\hline & $\beta(\operatorname{deg})$ & 33.9076 & 34.3695 & 0.0136223 \\
\hline & $\gamma(\operatorname{deg})$ & -0.952751 & -1.00388 & 0.0536646 \\
\hline & $t_{x}(\mathrm{~mm})$ & -317.854 & -316.238 & -0.00508409 \\
\hline & $t_{y}(\mathrm{~mm})$ & 0.795599 & 1.33328 & 0.675819 \\
\hline & $t_{z}(\mathrm{~mm})$ & 89.3097 & 108.485 & 0.214705 \\
\hline & line $(\mathrm{mm})$ & 330.163 & 334.331 & 0.0126241 \\
\hline
\end{tabular}

Based on the camera parameters calibrated at initial configuration and taking the first image pair as reference images, the surface U-, V- and W- displacement fields of the glass plate at various times can be computed. Figure 4 shows the corresponding virtual displacement fields of the last image pair recorded at 5 hours after initiating the image recording process. From the overall 3D displacement vector field shown in Fig.4 (a), it is seen that virtual 3D displacements have occurred to the test specimen surface. The 3D movement can be more clearly seen from the in-plane displacement vector field and the $\mathrm{W}$-displacement field indicated in Fig.4 (b) and (c), respectively. However, from the non-zero displacements and approximately evenly spaced contour lines clearly shown in Fig.4 (d) and (e), it can be further concluded that the specimen has undergone both virtual in-plane translations and virtual tensile strains. Since both the test reference object and the camera bodies were completely fixed, the reason for the image motions can only be attributed to the movements of the camera target sensors due to thermal expansion of the mechanical components in the two cameras. 

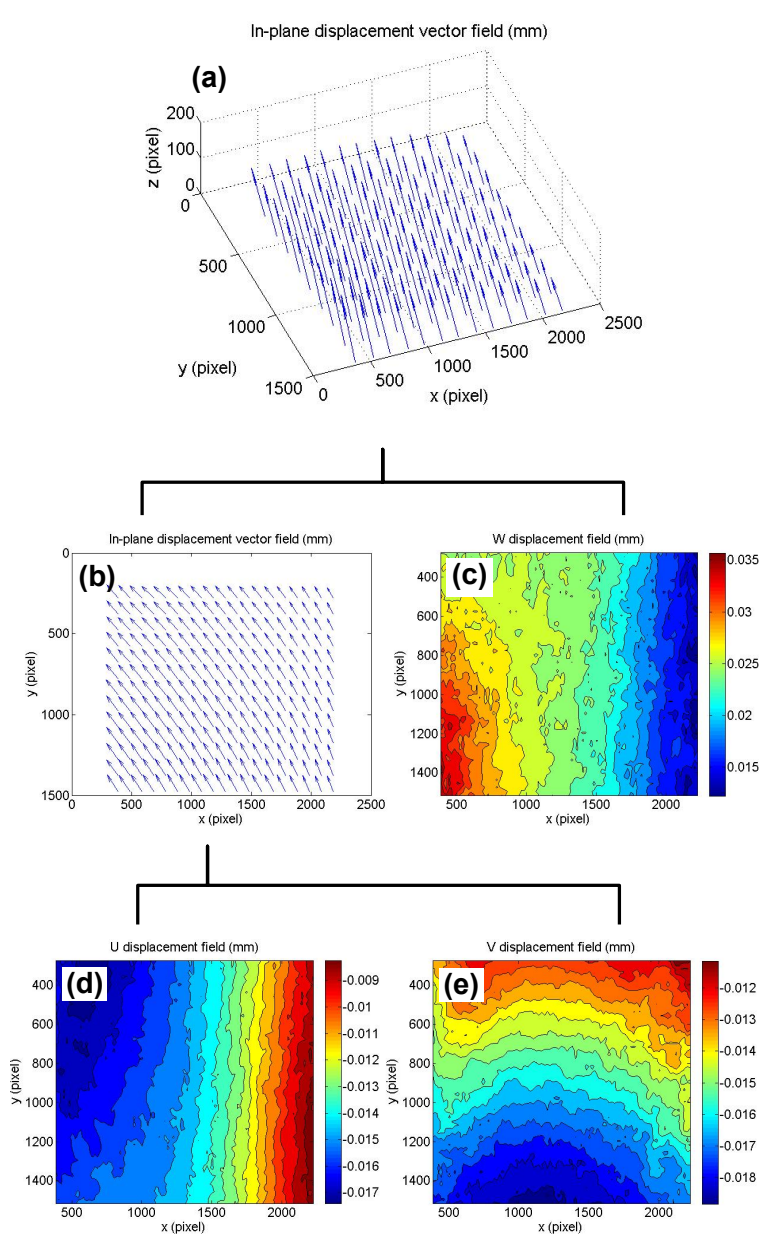

Fig. 4. The virtual displacement fields of quartz plane glass surface measured in the self-heating test: (a) 3D displacement vector field; (b) in-plane displacement vector field, (c) W-displacement field, (d) U-displacement field and (b) V-displacement field.

Based on the obtained displacement and strain fields at each time, the averaged displacement components $\mathrm{U}, \mathrm{V}, \mathrm{W}$ and average strain components $\varepsilon_{\mathrm{x}}, \varepsilon_{\mathrm{y}}, \gamma_{\mathrm{xy}}$ values can be computed for each image pairs. The results are plotted as a function of the recording time for the warm-up tests in Fig. 5. Similar to the results previously observed for single-camera 2D-DIC, both the virtual displacement and strain response due to self-heating of the digital cameras correlate with the detected temperature changes of the cameras, and can be separated into two consecutive stages. The first stage is a 1-2 hours warm-up stage after the camera was switched on. In this stage, the three displacement components, two normal strains and the camera bodies' temperature increase rapidly in almost the same way. After the warm-up stage of the cameras, the $\mathrm{U}, \mathrm{V}$ displacements stabilize at about -0.014 pixels, the $\mathrm{W}$ displacements fluctuate around 0.023 pixels. The two normal strains remain stable at $130 \mu \varepsilon$ as the cameras' temperatures keep constant, indicating a stain error of about $30 \mu \varepsilon /{ }^{\circ} \mathrm{C}$ (Here, it is necessary to note that the temperature change in the cameras between the first image pair and last image pair is about $4^{\circ} \mathrm{C}$ rather than $8^{\circ} \mathrm{C}$ ). Also, it is necessary to mention that the in-plane shear strains at various times are both close to zero, which indicate shear strains are not affected by temperature variations.
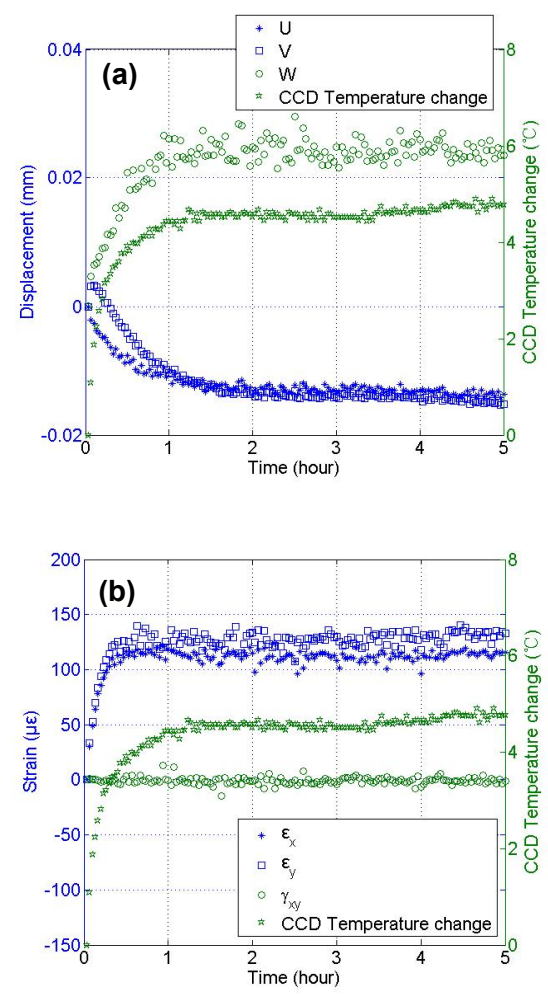

Fig. 5. The variations of (a) mean displacements and (b) mean strains over time during the camera self-heating experiment using the initially recorded calibration images and reference image pair.

Of course, by using the cameras' parameters calibrated after the image capture process and taking the last image pair as reference images, all the image pairs recorded before that time can be compared against the final one to compute their displacement fields and average strain components using the same analysis procedure. The corresponding averaged displacement components (i.e., $\mathrm{U}, \mathrm{V}$ and $\mathrm{W}$ ) and averaged strains (i.e., $\varepsilon_{\mathrm{x}}, \varepsilon_{\mathrm{y}}$ and $\gamma_{\mathrm{xy}}$ ) computed for each image pairs are plotted against the recording time for the same warm-up tests as shown in Fig. 6. Not surprisingly, it is observed that, for the image pairs recorded after 1 hour 
(when the cameras have reached thermal equilibrium), all these kinematic parameters approach zero. This implies that the stereo-DIC system is stable. However, for the image pairs recorded in the warm-up stage, displacements and strains with almost the same magnitudes but opposite signs are observed.
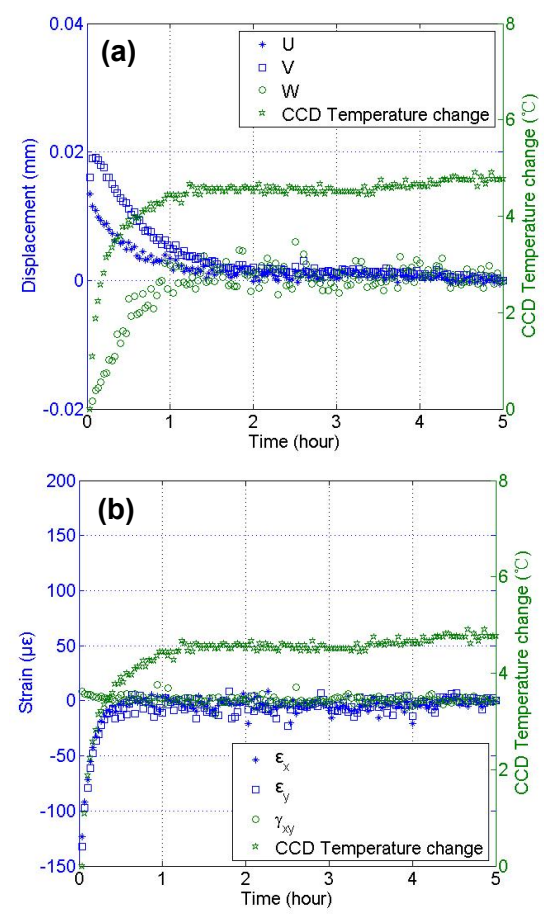

Fig.6. The variations of (a) mean displacements and (b) mean strains over time during the camera self-heating experiment using the finally recorded calibration images and final image pair as the reference state.

\section{B. Tests with varying ambient temperatures}

Figure 7(a) shows the temperature variations recorded by the 3 thermocouple sensors in the tests with varying ambient temperatures. With the aid of an air conditioner, the ambient temperatures were decreased from $30.2^{\circ} \mathrm{C}$ to about $25.9^{\circ} \mathrm{C}$ in the first stage, and then increased to $28.5^{\circ} \mathrm{C}$ in the second stage. Meanwhile the CCD temperature changed from $39.2^{\circ} \mathrm{C}$ to $35.8^{\circ} \mathrm{C}$, and then to $37.5^{\circ} \mathrm{C}$. The same temperature variation trends have been observed from the data recorded by the two thermocouples attached onto the cameras' body. However, the temperatures of the two cameras are about $9-10^{\circ} \mathrm{C}$ higher than ambient temperatures because of the self-heating effect of the cameras.

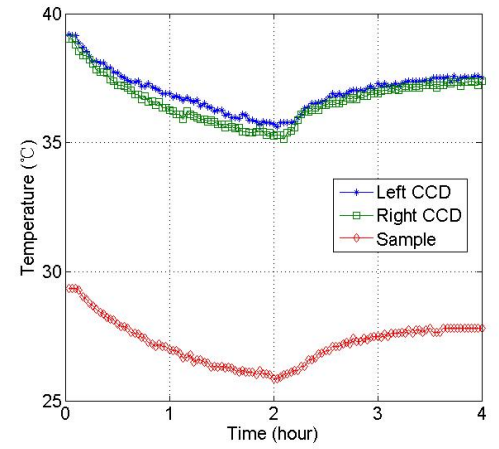

(a)

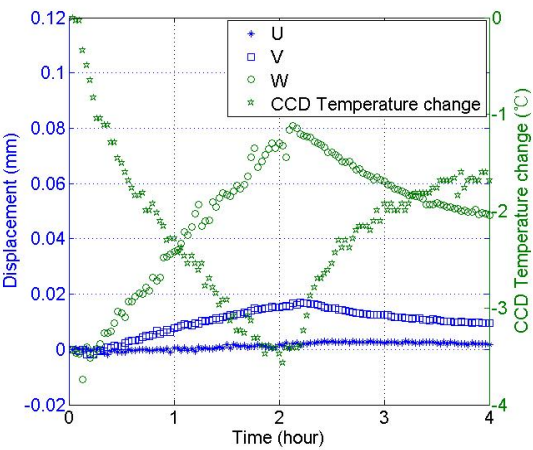

(b)

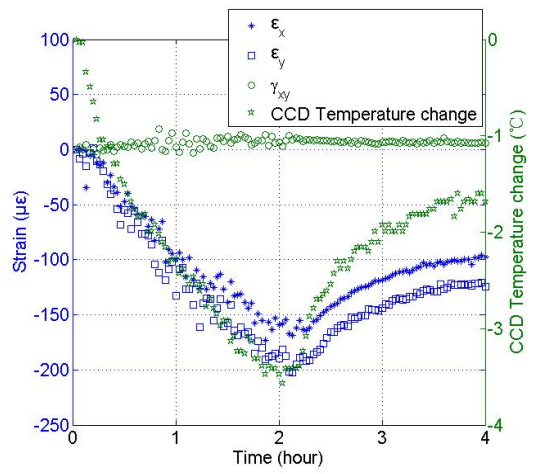

(c)

Fig.7. (a) The detected temperature variations of two cameras and the quartz glass plate, (b) the obtained mean displacements and (c) mean strains over time during the ambient temperature variation experiment

Using the initially recorded 30 calibration images after the cameras having obtained thermal equivalence, the camera parameters of the stereo-DIC system are retrieved. Also, take the initially recorded image pair before changing ambient temperature as reference images, the displacement fields and strain maps of the stationary quartz glass plate at various ambient temperatures can be calculated in a similar way we used in the previous subsection. Likewise, the averaged values of the three displacement components $(\mathrm{U}, \mathrm{V}$, W) and three in-plane strain components $\left(\varepsilon_{x}, \varepsilon_{y}, \gamma_{x y}\right)$ are 
estimated and plotted versus recording time in Fig.7(b) and (c). Note that in Figs. 7(b) and (c), the right label show the temperature variations of the CCD cameras relative to its initial state. Once again, it is observed that the average values of the virtual displacements and, in particular, strains measured by the stereo-DIC system also correlate with the detected temperature changes of the cameras. An inspection of Fig. 7(c) reveals that the magnitude of the temperature-induced normal stains is estimated as about $50 \mu \varepsilon /{ }^{\circ} \mathrm{C}$. Also, it is shown that the in-plane shear strains are not affected by temperature variations.

Figure 8 shows the virtual displacement fields of the image pair recorded at lowest ambient temperature. The almost evenly spaced contour lines shown in $\mathrm{U}$ and $\mathrm{V}$ fields shown Figs. 8 (a) and (b) imply virtual normal strains caused by ambient temperature variations. While for the W displacement fields, the displacement gradients are less pronounced. The observations are in good accordance with those shown in the self-heating tests. Again, the reason for these non-zero displacements is attributed to the movements of the camera target sensors due to thermal expansion of the mechanical components in the two cameras.
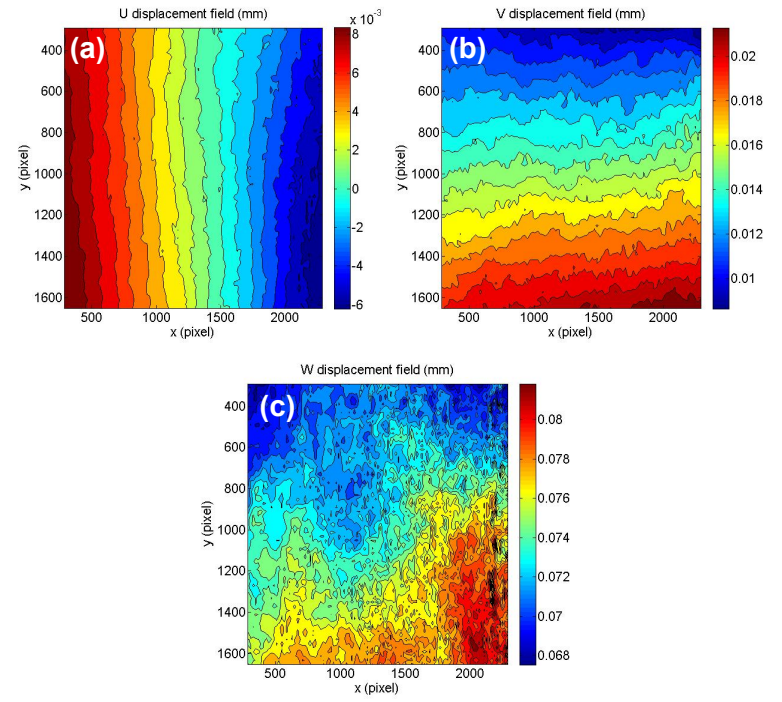

Fig.8. The virtual displacement fields of quartz plane glass surface measured in the ambient temperature variation experiment: (a) U-displacement field, (b) V-displacement field and (c) W-displacement field

\section{Conclusion and discussion}

In routine use of stereo-DIC system, static calibration model is implicitly adopted which generally relies on the calibration images captured prior to image recording process. However, the results of this work reveal that, if the cameras undergo temperature changes after initial calibration, the camera parameters of the stereo-DIC system changes accordingly when temperature varies, which in turn induce virtual displacements and strains highly correlated with temperature changes in the cameras. Since in most experimental mechanics applications, accuracy of stereo-DIC measurements is always the most important requirement, the virtual displacement and virtual strains due to temperature variations should be avoided or maximally eliminated.

Although it is possible to compensate the temperature-induced displacement errors associated with a specific stereo-DIC system by establishing the camera parameter-temperature model, we consider the numerical compensation approach may be two complicate and less practical Here we give two easy-to-implement but effective suggestions for minimizing the temperature-induced errors in practical stereo-DIC measurements:

(1) For indoor or short-term outdoor use of stereo-DIC technique, where it is relatively easy to keep the ambient temperature stable, we suggest that the stereo-DIC system should be fully preheated to reach its thermal equilibrium before recording calibration images and obtaining the reference and deformed images of the test specimen;

(2) For prolonged non-laboratory stereo-DIC applications where it may be difficult or impossible to keep the ambient temperature stable, the temperature-induced displacement and strain errors can be compensated using the reference specimen compensation approach we proposed recently [23]. Specifically, a fixed reference object without any mechanical and thermal deformation can be placed close to or adhered onto the test specimen to detect virtual displacement fields caused by temperature variations. Then, the virtual displacement fields can be fitted using linear polynomials. After that, the determined polynomial coefficients can be used to subtract the temperature-induced displacement from directly measured ones to recover the desired deformation fields.

\section{Acknowledgements:}

This work is supported by the National Natural Science 
Foundation of China (Grant nos. 11272032, 11322220, and 11427802), the Program for New Century Excellent Talents in University (Grant no. NCET-12-0023), the Science Fund of State Key Laboratory of Automotive Safety and Energy (Grant no. KF14032) and the Beijing Nova Program (xx2014B034). We also thank King Abdullah University of Science and Technology (KAUST) for its support.

\section{References:}

1. P.F. Luo, Y.J. Chao, M.A. Sutton, and W.H. Peters, "Accurate measurement of three-dimensional displacement in deformable bodies using computer vision.," Exp. Mech. 33(2), 123-132, (1993).

2. D. Garcia, J.J. Orteu, and L. Penazzi, “A combined temporal tracking and stereo-correlation technique for accurate measurement of 3D displacements: application to sheet metal forming," J. Mater. Process. Technol. 125, 736-742, (2002).

3. B. Pan, H.M. Xie, L. Yang, and Z.Y. Wang, "Accurate measurement of satellite antenna surface using 3D digital image correlation technique," Strain. 45(2), 194-200, (2009).

4. B. Pan, D.F. Wu, and L.P. Yu, "Optimization of a three-dimensional digital image correlation system for deformation measurements in extreme environments," Appl. Opt. 51(19), 4409-4419, (2012).

5. M. Malesa, K. Malowany, U. Tomczak, B. Siwek, M. Kujawińska, and A. Siemińska-Lewandowska, "Application of 3D Digital Image Correlation in maintenance and process control in industry," Comp. Indust. 64(9), 1301-1315, 2013.

6. “Correlated Solutions," http://www.correlatedsolutions.com.

7. “GOM Optical Measuring Techniques," http://www.gom.com.

8. http://www.pmlab.com.cn/

9. B. Pan, K. Li, and W. Tong, "Fast, robust and accurate digital image correlation calculation without redundant computations," Exp. Mech. 53(7), 1277-1289, (2013).

10. Y. Gao, T. Cheng, Y. Su, X.H. Xu, Y. Zhang, and Q.C. Zhang, "High-efficiency and high-accuracy digital image correlation for three-dimensional measurement," Opt. Lasers Eng. 65, 73-80 (2014).

11. B. Pan and L. Tian, "Superfast robust digital image correlation analysis using parallel computing," Opt. Eng. 54(3), 034106 (2015).

12. B. Pan, "Bias error reduction of digital image correlation using Gaussian pre-filtering," Opt. Lasers Eng. 51(10), 1161-1167 (2013).

13. Y.H. Zhou, C. Sun, and J.B. Chen, "Adaptive subset offset for systematic error reduction in incremental digital image correlation," Opt. Lasers Eng. 55, 5-11 (2014).
14. Z.Z. Tang, J. Liang, Z.Z. Xiao, C. Guo, and H. Hu, "Three-dimensional digital image correlation system for deformation measurement in experimental mechanics," Opt. Eng. 49(10), 103601 (2010).

15. M. Vo, Z.Y. Wang, L. Luu, and J. Ma, "Advanced geometric camera calibration for machine vision," Opt. Eng. 50(11), 110503-110503-3 (2011).

16. P. Reu, "Study of the Influence of Calibration Uncertainty on the Global Uncertainty for Digital Image Correlation Using a Monte Carlo Approach," Exp. Mech. 53, 1661-1680 (2013).

17. R. Reu, “Calibration: Stereo Calibration,” Exp. Tech. 38(1), 1-2, (2014).

18. H. Handel, "Analyzing the influences of camera warm-up effects on image acquisition. Computer Vision - ACCV 2007," Springer Berlin Heidelberg. 258-268 (2007).

19. S.P. Ma, J.Z. Pang, and Q.W. Ma, "The systematic error in digital image correlation induced by self-heating of a digital camera," Meas. Sci. Technol. 23(2), 025403 (2012).

20. B. Pan, L. P. Yu, and D.F. Wu, "High-accuracy 2D digital image correlation measurements with bilateral telecentric lenses: error analysis and experimental verification," Exp. Mech. 53(9), 1719-1733 (2013).

21. Q.F. Yu, Z.C. Chao, G.W. Jiang, Y. Shang, S.H. Fu, and et al., "The effects of temperature variation on videometric measurement and a compensation method," Image Vision Comput. 32(12), 1021-1029 (2014).

22. B. Pan, A. Asundi, H.M. Xie, and J.X. Gao, "Digital image correlation using iterative least squares and pointwise least squares for displacement field and strain field measurements," Opt. Lasers Eng. 47(7), 865-874 (2009).

23. B. Pan, L. P. Yu, and D.F. Wu, "High-accuracy 2D digital image correlation measurements using low-cost imaging lenses: implementation of a generalized compensation method," Meas. Sci. Technol. 25(2), 025001 (2014). 